\title{
Robust Filtering Under Randomly Varying Sensor Delay With Variance Constraints
}

\author{
Zidong Wang, Senior Member, IEEE, Daniel W. C. Ho, and Xiaohui Liu
}

\begin{abstract}
This paper deals with a new filtering problem for linear uncertain discrete-time stochastic systems with randomly varying sensor delay. The norm-bounded parameter uncertainties enter into the system matrix of the state space model. The system measurements are subject to randomly varying sensor delays, which often occur in information transmissions through networks. The problem addressed is the design of a linear filter such that, for all admissible parameter uncertainties and all probabilistic sensor delays, the error state of the filtering process is mean square bounded, and the steady-state variance of the estimation error for each state is not more than the individual prescribed upper bound. We show that the filtering problem under consideration can effectively be solved if there are positive definite solutions to a couple of algebraic Riccati-like inequalities or linear matrix inequalities. We also characterize the set of desired robust filters in terms of some free parameters. An illustrative numerical example is used to demonstrate the usefulness and flexibility of the proposed design approach.
\end{abstract}

Index Terms-Algebraic matrix inequality, Kalman filtering, parameter uncertainty, random sensor delay, robust filtering.

\section{INTRODUCTION}

I $\mathrm{N}$ CLASSICAL Kalman minimum variance filtering, it is implicitly assumed that the signal models are exactly known, and the noise sources are stationary-white-noise signals with known statistics. Unfortunately, these assumptions limit the application of minimum variance filters as in many situations only an approximate signal model is available and/or the statistics of the noise sources are not fully known or unavailable. To handle the above problem, in recent years, two alternative design methods have been developed, namely, the $H_{\infty}$ filtering and the robust filtering approaches, see for example [4], [6], [9], [13] and references therein. The main point of the $H_{\infty}$ filtering theory is to design an estimator that ensures a bound on the induced $L_{2}$-norm of the operator from the noise signals to the estimation error, while the robust filtering theory aims at guaranteeing an upper bound to the quadratic cost (i.e., estima-

Manuscript received May 17, 2002; revised September 15, 2003. This work was supported in part by the EPSRC under Grant GR/S27658/01 and Grant GR/R35018/01, in part by the Nuffield Foundation under Grant NAL/00630/G, in part by the Research Grants Council of Hong Kong SAR under Grant CityU/101103, in part by the City University of Hong Kong under Grant CityU/SRG/7001146, and in part by the Alexander von Humboldt Foundation of Germany. This paper was recommended by Associate Editor M. Pedram.

Z. Wang and X. Liu are with the Department of Information Systems and Computing, Brunel University, Uxbridge UB8 3PH, U.K. (e-mail: Zidong.Wang@brunel.ac.uk).

D. W. C. Ho is with the Department of Mathematics, City University of Hong Kong, Hong Kong.

Digital Object Identifier 10.1109/TCSII.2004.829572 tion-error variance) in spite of various parameter uncertainties, and subsequently minimizing this upper bound locally.

In many practical filtering problems, such as the tracking of a maneuvering target and data fusion subject to highly stochastic disturbances, the filtering performance requirements are often described in terms of the upper bounds on the error variances of estimation, see, e.g., [16], [17], [21]. Note that, here, the steady-state error variance is not required to be the minimum, but should not be more than the specified upper bound constraint. The conventional filtering methods, however, are usually difficult to be applied in dealing with this class of constrained variance filtering problems. For instance, the theory of weighted least-squares estimation ([14]) minimizes a weighted scalar sum of the error variances of the state estimation, but minimizing a scalar sum does not ensure that the multiple variance requirements will be satisfied. The so-called error covariance assignment (ECA) theory was initiated in [21] to provide a closed form solution for directly placing the specified steady-state estimation error covariance to a linear system. Subsequently, based on the ECA theory, a more general filtering method, namely, variance-constrained filtering method, has been developed, and applied to some more realistic systems, such as parameter uncertain systems [16], sampled-data systems [17], and bilinear systems [18]. With respect to the variance-constrained filtering approach, after assigning to the system a specified variance upper bound, there still remains mисh freedom, which can be used to attempt to directly achieve other desired performance requirements, but the traditional optimal (robust and/or $H_{\infty}$ ) Kalman filtering methods may not be of such an advantage.

On the other hand, besides the system parameter uncertainties, it has been well recognized that the time delay is also often the main cause of instability and poor performance of systems [7]. In the past few years, the filter design problems for uncertain time-delay systems have begun to receive research attention, see, e.g., [10], [19]. So far, in most relevant literature, the time-delay is always assumed to be deterministic. However, for a large class of practical applications, the time delay may occur in a random way. A typical case is the network-based information transmission process. In such a case, random delay is induced by an asynchronous time-division-multiplexed network that serves as a data communications link between the spatially dispersed components of the integrated "decision" and "control" systems [11]. Examples include the signal receiving process of a mobile phone based in a network, the vehicle management system of future generation aircraft, etc., where the sensor (receiver) data is subjected to randomly varying delays induced by the network before they arrive at their respective 
destinations. In this sense, new filtering methods should be designed to meet the needs for signal processing in a delayed environment of network-based systems.

It should be pointed out that, if the system measurements contain random time-delays, the noise statistics cannot be exactly known, and subsequently the traditional robust and/or $H_{\infty}$ filtering approaches are not applicable to this case. So far, there have been very few results appeared to cope with the filter design problem for parameter uncertain system having randomly delayed outputs. An error covariance assignment method was developed in [22] for the state estimation problem of linear random models with sensor delay, but the system uncertainty (modeling error) was not taken into account. On the other hand, due to the appearance of both the parameter uncertainty and random delay, the use of popular minimum variance filtering approach seems very difficult. Instead, the constrained variance filtering method might be more practical because of its flexibility. Up to now, to the best of the authors' knowledge, the issue of variance-constrained filtering on parameter uncertain systems with randomly varying sensor delay has not been fully investigated and remains to be important and challenging.

In this paper, we are concerned with the filter design problem for linear uncertain discrete-time stochastic systems with randomly varying sensor delay. The parameter uncertainties are allowed to be time-varying and norm-bounded, and appear in the system state matrix. The system measurements are subject to randomly varying sensor delays, which often occur in information transmission through network. We are interested in designing linear filters such that, for all admissible parameter uncertainties and all probabilistic sensor delays, the error state of the filtering process is mean square bounded, and the steadystate variance of the estimation error of each state is not more than the individual prescribed upper bound. We show that the filtering problem under consideration can effectively be solved if there are positive definite solutions to a couple of algebraic Riccati-like inequalities or linear matrix inequalities. We also characterize the set of desired robust filters in terms of some free parameters. An illustrative numerical example is used to demonstrate the usefulness and flexibility of the proposed design approach.

The rest of this paper is arranged as follows. Section II formulates the robust variance-constrained filter design problem for uncertain discrete-time systems with random sensor delay. Some preliminary results are given in Section III, and the solution of the addressed problem is provided in Section IV. We demonstrate the use of the proposed theory in Section V by means of a numerical example. Some concluding remarks are drawn in Section VI.

Notation. The notations in this paper are quite standard. $\mathbb{R}^{n}$ and $\mathbb{R}^{n \times m}$ denote, respectively, the $n$ dimensional Euclidean space and the set of all $n \times m$ real matrices. The superscript " $T$ " denotes the transpose and the notation $X \geq Y$ (respectively, $X>Y$ ) where $X$ and $Y$ are symmetric matrices, means that $X-Y$ is positive semi-definite (respectively, positive definite). $I$ is the identity matrix with compatible dimension. $A^{+}$stands for the Moore-Penrose inverse of a matrix $A$. Let $\left(\Omega, \mathcal{F},\left\{\mathcal{F}_{t}\right\}_{t \geq 0}, P\right)$ be a complete probability space with a filtration $\left\{\mathcal{F}_{t}\right\}_{t \geq 0}$ satisfying the usual conditions (i.e., the filtration contains all $P$-null sets and is right continuous). $\mathcal{E}\{\cdot\}$ stands for the mathematical expectation operator with respect to the given probability measure $P$. Prob $\{\cdot\}$ means the occurrence probability of the event ".." The shorthand $\operatorname{diag}\left(M_{1}, M_{2}, \ldots, M_{N}\right)$ denotes a block diagonal matrix with diagonal blocks being the matrices $M_{1}, M_{2}, \ldots, M_{N}$. Sometimes, the arguments of a function will be omitted in the analysis when no confusion can arise.

\section{Problem Formulation AND PRELIMINARIES}

Let the digital signal be generated from the following linear uncertain discrete-time stochastic system

$$
\tilde{x}(k+1)=(\tilde{A}+\Delta \tilde{A}(k)) \tilde{x}(k)+w(k)
$$

and the delayed sensor model be described by

$$
\begin{aligned}
& \tilde{y}(k)=\tilde{C} \tilde{x}(k)+\tilde{v}(k) \\
& y(k)=(1-\gamma(k)) \tilde{y}(k)+\gamma(k) \tilde{y}(k-1)
\end{aligned}
$$

where $\tilde{x} \in \mathbb{R}^{n}$ is a state vector, $y \in \mathbb{R}^{p}$ is an actual measured output vector, and $\tilde{A}$ and $\tilde{C}$ are known constant matrices. $w(k) \in \mathbb{R}^{n}$ and $\tilde{v}(k) \in \mathbb{R}^{p}$ are mutually uncorrelated zero mean Gaussian white-noise sequences with respective covariance $W>0$ and $\tilde{V}>0$. The initial state $\tilde{x}(0)$ has the mean $\overline{\tilde{x}}(0)$ and covariance $P(0)$, and is uncorrelated with both $w(k)$ and $\tilde{v}(k) . \Delta \tilde{A}(k)$ is a real-valued uncertain matrix satisfying

$$
\Delta \tilde{A}(k)=\tilde{M} F(k) \tilde{N}, \quad F(k) F^{T}(k) \leq I
$$

and $\tilde{M}$ and $\tilde{N}$ are known constant matrices of appropriate dimensions, which specify how the elements of the nominal matrix $\tilde{A}$ are affected by the uncertain parameters in $F$. The parameter uncertainties in $\Delta \tilde{A}$ are said to be admissible if (4) holds.

The stochastic variable $\gamma(k) \in \mathbb{R}$ is a Bernoulli distributed white sequence taking values on 0 and 1 with

$$
\operatorname{Prob}\{\gamma(k)=1\}=\mathcal{E}\{\gamma(k)\}:=\bar{\gamma}
$$

where $\bar{\gamma} \in \mathbb{R}$ is a known constant, and $\gamma(k) \in \mathbb{R}$ is assumed to be independent of $w(k), \tilde{v}(k)$, and $\tilde{x}(0)$. Therefore, we have

$$
\begin{aligned}
\operatorname{Prob}\{\gamma(k) & =0\}=1-\bar{\gamma} \\
\sigma_{\tilde{\gamma}}^{2} & :=\mathcal{E}\left\{(\gamma(k)-\bar{\gamma})^{2}\right\}=(1-\bar{\gamma}) \bar{\gamma} .
\end{aligned}
$$

Remark 1: The time-varying parameter uncertainty structure as in (4) has been frequently used in the problems of robust filtering and control of uncertain systems (see, e.g., [16]). The system measurement mode (3) was introduced in [22], which can be used to represent the system output subject to randomly varying state delay. It is observed that, at $k$ th sampling time, the actual system output takes the value $\tilde{y}(k-1)$ with probability $\bar{\gamma}$, and the value $\tilde{y}(k)$ with probability $1-\bar{\gamma}$. The corresponding scalar $\bar{\gamma}(0<\bar{\gamma}<1)$ could be estimated through statistical tests, and some related discussions can be found in [20].

Throughout the paper, the following assumption is made.

Assumption 1: The matrix $\tilde{A}$ is Schur stable (i.e., all eigenvalues of $\tilde{A}$ are located within the unit circle in the complex plane). 
By defining

$$
\begin{aligned}
x(k) & :=\left[\begin{array}{c}
\tilde{x}(k) \\
\tilde{x}(k-1)
\end{array}\right] \\
A & :=\left[\begin{array}{cc}
\tilde{A} & 0 \\
I_{n} & 0
\end{array}\right] \\
M & :=\left[\begin{array}{c}
\tilde{M} \\
0
\end{array}\right] \\
N & :=\left[\begin{array}{ll}
\tilde{N} & 0
\end{array}\right] \\
\Delta A(k) & :=M F(k) N \\
C(\gamma(k)) & =\left[\begin{array}{ll}
(1-\gamma(k)) \tilde{C} & \gamma(k) \tilde{C}
\end{array}\right] \\
D_{1} & =\left[\begin{array}{c}
I_{n} \\
0
\end{array}\right] \\
D_{2}(\gamma(k)) & =\left[\begin{array}{ll}
(1-\gamma(k)) I_{p} & \gamma(k) I_{p}
\end{array}\right] \\
v(k) & =\left[\begin{array}{c}
\tilde{v}(k) \\
\tilde{v}(k-1)
\end{array}\right] .
\end{aligned}
$$

A compact representation of the system (1)-(3) can be given as follows:

$$
\begin{aligned}
x(k+1) & =(A+\Delta A(k)) x(k)+D_{1} w(k) \\
y(k) & =C(\gamma(k)) x(k)+D_{2}(\gamma(k)) v(k)
\end{aligned}
$$

where $v(k)$ is a zero mean Gaussian white-noise sequence with covariance

$$
V:=\left[\begin{array}{cc}
\tilde{V} & 0 \\
0 & \tilde{V}
\end{array}\right]
$$

and is independent of $w(k), \gamma(k)$, and $x(0)$. Note that $C(\gamma(k))$ and $D_{2}(\gamma(k))$ are functions of the stochastic variable $\gamma(k)$, and therefore, (12)-(13) is in fact a stochastic parameter system.

Define

$$
\bar{C}=\mathcal{E}[C(\gamma(k))]=[(1-\bar{\gamma}) \tilde{C} \bar{\gamma} \tilde{C}] .
$$

The linear full-order filter considered in this paper is of the following structure:

$$
\hat{x}(k+1)=G \hat{x}(k)+K(y(k)-\bar{C} \hat{x}(k))
$$

where $\hat{x}(k) \in \mathbb{R}^{2 n}$ means that the state estimate of the stochastic parameter system (12)-(13), and the constant matrices $G$ and $K$ are filter parameters to be scheduled.

The steady-state estimation error covariance is defined by

$$
\begin{aligned}
& X_{e e}:=\lim _{k \rightarrow \infty} X_{e e}(k):=\lim _{k \rightarrow \infty} \mathcal{E}\left[e(k) e^{T}(k)\right] \\
& e(k)=x(k)-\hat{x}(k) .
\end{aligned}
$$

The purpose of this paper is to design the filter parameters $G$ and $K$ such that, for all admissible parameter perturbations in $\Delta \tilde{A}$ and all randomly varying sensor delays: 1) the matrix sequence $X_{e e}(k)$ is convergent; and 2) the steady-state value $X_{e e}$ of the sequence $X_{e e}(k)$ satisfies

$$
\left[X_{e e}\right]_{i i} \leq \alpha_{i}^{2}, \quad i=1,2, \ldots, 2 n
$$

where $\left[X_{e e}\right]_{i i}$ means the steady-state variance of the $i$ th error state and $\alpha_{i}^{2}(i=1,2, \ldots, 2 n)$ denotes the prespecified steadystate error-estimation variance constraint on the $i$ th state.

\section{SOME PRELIMINARY RESUltS}

Now introducing a new stochastic sequence

$$
C^{e}(\gamma(k)):=C(\gamma(k))-\bar{C}
$$

we can see that $C^{e}(\gamma(k)) \in \mathbb{R}^{p \times 2 n}$ is a zero mean stochastic matrix sequence. Then, we obtain from (12)-(15) and (17) that

$$
\begin{aligned}
y(k)-\bar{C} \hat{x}(k) & =C(\gamma(k)) x(k)+D_{2}(\gamma(k)) v(k)-\bar{C} \hat{x}(k) \\
& =C^{e}(\gamma(k)) x(k)+\bar{C} e(k)+D_{2}(\gamma(k)) v(k)
\end{aligned}
$$

and hence

$$
\begin{aligned}
e(k+1)= & {\left[A+\Delta A-G-K C^{e}(\gamma(k))\right] x(k)+(G-K \bar{C}) } \\
& \cdot e(k)+D_{1} w(k)-K D_{2}(\gamma(k)) v(k) .
\end{aligned}
$$

Again, for convenience of later analysis, we define

$$
\begin{aligned}
x_{f}(k) & :=\left[\begin{array}{c}
x(k) \\
e(k)
\end{array}\right] \\
A_{f} & :=\left[\begin{array}{cc}
A & 0 \\
A-G-K C^{e}(\gamma(k)) & G-K \bar{C}
\end{array}\right] \\
M_{f} & :=\left[\begin{array}{c}
M \\
M
\end{array}\right] \\
N_{f} & :=\left[\begin{array}{ll}
N & 0
\end{array}\right] \\
\Delta A_{f} & =M_{f} F N_{f} \\
w_{f}(k) & :=\left[\begin{array}{ll}
D_{1} w(k)-K D_{2}(\gamma(k)) v(k)
\end{array}\right] \\
X(k) & :=\mathcal{E}\left[x_{f}(k) x_{f}^{T}(k)\right]:=\left[\begin{array}{cc}
X_{x x}(k) & X_{x e}(k) \\
X_{x e}^{T}(k) & X_{e e}(k)
\end{array}\right] .
\end{aligned}
$$

Considering (12) and (20), we obtain the following augmented system:

$$
x_{f}(k+1)=\left(A_{f}+\Delta A_{f}\right) x_{f}(k)+w_{f}(k)
$$

where $w_{f}(k)$ denotes a zero-mean Gaussian white-noise sequence.

Remark 2: It is mentionable that there are stochastic sequences $C^{e}(\gamma(k))$ and $D_{2}(\gamma(k))$ involved in $A_{f}$ and $w_{f}(k)$, respectively. This makes the augmented system (25) a stochastic parameter one, which reflects the characteristic of randomly varying sensor delays. Notice that in the literature, the robust filtering problem for stochastic parameter systems has not gained much attention mainly because of the complexity of the statistics analysis.

We first analyze the second-order information of the augmented noise $w_{f}(k)$. From the statistical property of $\gamma(k)$, the definition (11), and the relation $V=\operatorname{diag}\{\tilde{V}, \tilde{V}\}$, we have

$$
\begin{aligned}
\mathcal{E}\left[D_{2}(\gamma(k)) V D_{2}^{T}(\gamma(k))\right] & =\mathcal{E}\left[(1-\gamma(k))^{2} \tilde{V}+\gamma^{2}(k) \tilde{V}\right] \\
& =(1-\bar{\gamma}) \tilde{V}+\bar{\gamma} \tilde{V}=\tilde{V}
\end{aligned}
$$

and hence

$$
\begin{aligned}
W_{f} & :=\mathcal{E}\left[w_{f}(k) w_{f}^{T}(k)\right] \\
& =\left[\begin{array}{cc}
D_{1} W D_{1}^{T} & D_{1} W D_{1}^{T} \\
D_{1} W D_{1}^{T} & D_{1} W D_{1}^{T}+K \tilde{V} K^{T}
\end{array}\right] .
\end{aligned}
$$


Now, noticing that $X_{x x}(k)=\operatorname{diag}\{\tilde{X}(k) \tilde{X}(k-1)\}$, where $\tilde{X}(k):=\mathcal{E}\left[\tilde{x}(k) \tilde{x}^{T}(k)\right]$, we have

$$
\begin{aligned}
& \mathcal{E}\left[C^{e}(\gamma(k)) X_{x x}(k)\left(C^{e}(\gamma(k))\right)^{T}\right] \\
& =\mathcal{E}\left[(\bar{\gamma}-\gamma(k))^{2}\left(\tilde{C} \tilde{X}(k) \tilde{C}^{T}+\tilde{C} \tilde{X}(k-1) \tilde{C}^{T}\right)\right] \\
& =\bar{\gamma}(1-\bar{\gamma})\left[\tilde{C} \tilde{X}(k) \tilde{C}^{T}+\tilde{C} \tilde{X}(k-1) \tilde{C}^{T}\right] \\
& =\bar{\gamma}(1-\bar{\gamma})\left[\begin{array}{ll}
\tilde{C} & \tilde{C}
\end{array}\right] X_{x x}(k)\left[\begin{array}{ll}
\tilde{C} & \tilde{C}
\end{array}\right]^{T} .
\end{aligned}
$$

Furthermore, considering (28) and using the statistics of the noises $w(k), v(k)$ and, in particular, $C^{e}(\gamma(k)), X(k)$ defined in (24) is found to satisfy

$X(k+1)=\left(A_{n}+\Delta A_{f}\right) X(k)\left(A_{n}+\Delta A_{f}\right)^{T}+J X J^{T}+W_{f}$

where

$$
\begin{aligned}
& A_{n}:=\left[\begin{array}{cc}
A & 0 \\
A-G & G-K \bar{C}
\end{array}\right] \\
& J:=\left[\begin{array}{ccc}
0 & & 0 \\
(\bar{\gamma}(1-\bar{\gamma}))^{1 / 2} K\left[\begin{array}{ll}
\tilde{C} & \tilde{C}
\end{array}\right] & 0
\end{array}\right]
\end{aligned}
$$

and $W_{f}$ is defined in (27).

We know from [1] and [3] that, if the state of (25) is meansquare bounded, then, the steady-state covariance $X$ of (25) defined by

$$
X:=\lim _{k \rightarrow \infty} X(k)=\left[\begin{array}{cc}
X_{x x} & X_{x e} \\
X_{x e}^{T} & X_{e e}
\end{array}\right]
$$

exists and satisfies the following discrete-time modified Lyapunov equation:

$$
X=\left(A_{n}+\Delta A_{f}\right) X\left(A_{n}+\Delta A_{f}\right)^{T}+J X J^{T}+W_{f} .
$$

Remark 3: It also follows from [1] and [3] that there exists a unique symmetric positive semi-definite solution to (32) if and only if

$$
\rho\left\{\left(A_{n}+\Delta A_{f}\right) \otimes\left(A_{n}+\Delta A_{f}\right)+J \otimes J\right\}<1
$$

where $\rho$ is the spectral radius and $\otimes$ is the Kronecker product. Furthermore, we know from [1] and [3] that the condition (33) is equivalent to the mean square boundedness of the state of the system (25). Hence, we conclude that, if there exists a positive definite solution to the (32), then, (33) holds and the convergence of $X(k)$ in (24) is guaranteed to a constant value $X$.

The purpose of this paper, which has been given in Section II, can now be restated as follows: to design the filter parameters $G$ and $K$, such that, for all admissible parameter uncertainties and all probabilistic sensor delays: 1) the augmented system (25) is mean-square bounded, i.e., (33) holds; and 2) the steady-state error covariance $X_{e e}$ satisfies

$$
\left[X_{e e}\right]_{i i} \leq \alpha_{i}^{2}, \quad i=1,2, \ldots, 2 n
$$

\section{MAIN Results AND PROOFS}

In this section, we shall first characterize an upper bound on the steady-state error covariance $X$ satisfying (32) in terms of some free parameters, and let this upper bound meet the prespecified variance constraints (34). Then, we shall parameterize all desired filter gains with which the resulting steady-state error covariance is not more than the obtained upper bound.

To start with, we recall the following lemmas that will be needed in the proof of our main results.

Lemma 1: [2]: Given matrices $\Xi \in \mathbb{R}^{s \times r}$ and $\Upsilon \in \mathbb{R}^{s \times t}$. There exists a solution $X \in \mathbb{R}^{r \times t}$ to the matrix equation $\Xi X=$ $\Upsilon$ if and only if $\left(I-\Xi \Xi^{+}\right) \Upsilon=0$, where $\Xi^{+}$denotes the Moore-Penrose inverse of $\Xi$. Furthermore, all solutions can be parameterized by

$$
X=\Xi^{+} \Upsilon+\left(I-\Xi^{+} \Xi\right) Z
$$

where $Z \in \mathbb{R}^{r \times t}$ is an arbitrary matrix.

Lemma 2: [15]: Let a positive scalar $\varepsilon>0$ and a positive definite matrix $Q_{f}>0$ be such that $N_{f} Q_{f} N_{f}^{T}<\varepsilon I$, and $\Delta A_{f}=M_{f} F(k) N_{f}$ with $F(k) F^{T}(k) \leq I$. Then

$$
\begin{aligned}
& \left(A_{n}+\Delta A_{f}\right) Q_{f}\left(A_{n}+\Delta A_{f}\right)^{T} \\
& \quad \leq A_{n}\left(Q_{f}^{-1}-\varepsilon^{-1} N_{f}^{T} N_{f}\right)^{-1} A_{n}^{T}+\varepsilon M_{f} M_{f}^{T}
\end{aligned}
$$

holds for all admissible perturbations $\Delta A$.

Lemma 3: [16]: For a given negative definite matrix $\Pi<$ $0\left(\Pi \in \mathbb{R}^{2 n \times 2 n}\right)$, there always exists a matrix $L \in \mathbb{R}^{2 n \times p}(p \leq$ $2 n$ ) such that $\Pi+L L^{T}<0$.

Lemma 4: (Schur Complement): Given constant matrices $\Omega_{1}, \Omega_{2}, \Omega_{3}$ where $\Omega_{1}=\Omega_{1}^{T}$ and $0<\Omega_{2}=\Omega_{2}^{T}$, then, $\Omega_{1}+\Omega_{3}^{T} \Omega_{2}^{-1} \Omega_{3}<0$ if and only if

$$
\left[\begin{array}{cc}
\Omega_{1} & \Omega_{3}^{T} \\
\Omega_{3} & -\Omega_{2}
\end{array}\right]<0 \quad \text { or } \quad\left[\begin{array}{cc}
-\Omega_{2} & \Omega_{3} \\
\Omega_{3}^{T} & \Omega_{1}
\end{array}\right]<0
$$

Lemma 5: (Matrix Inverse Lemma): Let $A, B, C$ and $D$ be given matrices of appropriate dimension with $A, D$, and $D^{-1}+$ $C A^{-1} B$ being invertible, then

$$
(A+B D C)^{-1}=A^{-1}-A^{-1} B\left(D^{-1}+C A^{-1} B\right)^{-1} C A^{-1} .
$$

For presentation convenience, we define

$$
\begin{aligned}
\Phi:= & (A-G)\left(P_{1}^{-1}-\varepsilon^{-1} N^{T} N\right)^{-1}(A-G)^{T} \\
& +\varepsilon M M^{T}+D_{1} W D_{1}^{T} \\
\hat{C}:= & {\left[\begin{array}{ll}
\tilde{C} & \tilde{C}
\end{array}\right] } \\
\Lambda:= & A\left(P_{1}^{-1}-\varepsilon^{-1} N^{T} N\right)^{-1} \\
R:= & \bar{\gamma}(1-\bar{\gamma}) \hat{C} P_{1} \hat{C}^{T}+\bar{C} P_{2} \bar{C}^{T}+\tilde{V} \\
\Pi:= & \Phi+G P_{2} G^{T}-P_{2}-G P_{2} \bar{C}^{T} R^{-1} \bar{C} P_{2} G^{T} .
\end{aligned}
$$

It is shown in the following theorem that the solution to the problem of variance-constrained filtering with random sensor delay is related to several quadratic matrix inequalities. 
Theorem 1: Assume that there exists a positive scalar $\varepsilon$ such that the following two quadratic matrix inequalities:

$$
\begin{aligned}
& A P_{1} A^{T}-P_{1}+A P_{1} N^{T}\left(\varepsilon I-N P_{1} N^{T}\right)^{-1} N P_{1} A^{T} \\
& \quad+\varepsilon M M^{T}+D_{1} W D_{1}^{T}<0 \\
& \Pi=\Phi+G P_{2} G^{T}-P_{2}-G P_{2} \bar{C}^{T} R^{-1} \bar{C} P_{2} G^{T}<0
\end{aligned}
$$

respectively, have positive definite solutions $P_{1}$ and $P_{2}$, and also $\varepsilon$ and $P_{1}$ satisfy $N P_{1} N^{T} \leq \varepsilon I$ and

$$
\left(I-\Lambda \Lambda^{+}\right)\left(\varepsilon M M^{T}+D_{1} W D_{1}^{T}\right)=0
$$

where $\Lambda^{+}$denotes the Moore-Penrose inverse of $\Lambda$, and in (42)

$$
G=A+\left[\Lambda^{+}\left(\varepsilon M M^{T}+D_{1} W D_{1}^{T}\right)\right]^{T} .
$$

Moreover, let $L \in \mathbb{R}^{2 n \times p}(p \leq 2 n)$ be an arbitrary matrix satisfying $\Pi+L L^{T}<0$ (see Lemma 3 ), and $U \in \mathbb{R}^{p \times p}$ be an arbitrary orthogonal matrix (i.e., $U U^{T}=I$ ). Then, the filter (16) with the parameters determined by (44) and

$$
K=G P_{2} \bar{C}^{T} R^{-1}+L U R^{-1 / 2}
$$

will be such that, for all admissible parameter uncertainties in $\Delta A$ and random sensor delays: 1) the state of the augmented system (25) is mean-square bounded; and 2) the steady-state error covariance $X_{e e}$ meets $X_{e e}<P_{2}$.

Proof: Define $P_{f}:=\operatorname{diag}\left(P_{1}, P_{2}\right)$. Then, it follows directly from Lemma 2 and the definitions (36)-(40) that

$$
\begin{aligned}
& \left(A_{n}+\Delta A_{f}\right) P_{f}\left(A_{n}+\Delta A_{f}\right)^{T}-P_{f}+J P_{f} J^{T}+W_{f} \\
\leq & A_{n}\left(P_{f}^{-1}-\varepsilon^{-1} N_{f}^{T} N_{f}\right)^{-1} A_{n}^{T} \\
& +\varepsilon M_{f} M_{f}^{T}-P_{f}+J P_{f} J^{T}+W_{f} \\
: & =\Psi:=\left[\begin{array}{ll}
\Psi_{11} & \Psi_{12} \\
\Psi_{12}^{T} & \Psi_{22}
\end{array}\right]
\end{aligned}
$$

where

$$
\begin{aligned}
\Psi_{11}= & A\left(P_{1}^{-1}-\varepsilon^{-1} N^{T} N\right)^{-1} A^{T}-P_{1}+\varepsilon M M^{T} \\
& +D_{1} W D_{1}^{T} \\
\Psi_{12}= & A\left(P_{1}^{-1}-\varepsilon^{-1} N^{T} N\right)^{-1}(A-G)^{T} \\
& +\varepsilon M M^{T}+D_{1} W D_{1}^{T} \\
\Psi_{22}= & (A-G)\left(P_{1}^{-1}-\varepsilon^{-1} N^{T} N\right)^{-1}(A-G)^{T} \\
& +(G-K \bar{C}) P_{2}(G-K \bar{C})^{T} \\
& +\varepsilon M M^{T}-P_{2}+\bar{\gamma}(1-\bar{\gamma}) K \hat{C} P_{1} \hat{C}^{T} K^{T} \\
& +D_{1} W D_{1}^{T}+K \tilde{V} K^{T} .
\end{aligned}
$$

It follows immediately from Lemma 5 that

$\left(P_{1}^{-1}-\varepsilon^{-1} N^{T} N\right)^{-1}=P_{1}+P_{1} N^{T}\left(\varepsilon I-N P_{1} N^{T}\right)^{-1} N P_{1}$

and therefore, the inequality (41) implies that $\Psi_{11}<0$.

Next, notice that the matrix $A$ is singular. It then follows from Lemma 1 that, there exists a solution $G$ such that $\Psi_{12}=0$ if and only if (43) holds. Furthermore, if (43) is true, (44) gives a solution. Hence, substituting the expression of $G$ in (44) into (48) leads to $\Psi_{12}=0$ easily.
Moreover, we now consider $\Psi_{22}$. By using the definitions (36)-(40), we can rearrange (49) as follows:

$$
\begin{aligned}
\Psi_{22}= & \Phi+(G-K \bar{C}) P_{2}(G-K \bar{C})^{T}-P_{2} \\
& +\bar{\gamma}(1-\bar{\gamma}) K \hat{C} P_{1} \hat{C}^{T} K^{T}+K \tilde{V} K^{T} \\
= & \Phi+G P_{2} G^{T}-P_{2}+K \\
& \times\left[\bar{\gamma}(1-\bar{\gamma}) \hat{C} P_{1} \hat{C}^{T}+\bar{C} P_{2} \bar{C}^{T}+\tilde{V}\right] K^{T} \\
& -G P_{2} \bar{C}^{T} K^{T}-K \bar{C} P_{2} G^{T} \\
= & \Phi+G P_{2} G^{T}-P_{2}-G P_{2} \bar{C}^{T} R^{-1} \bar{C} P_{2} G^{T} \\
& +\left(K R^{1 / 2}-G P_{2} \bar{C}^{T} R^{-1 / 2}\right) \\
& \times\left(K R^{1 / 2}-G P_{2} \bar{C}^{T} R^{-1 / 2}\right)^{T} \\
= & \Pi+\left(K R^{1 / 2}-G P_{2} \bar{C}^{T} R^{-1 / 2}\right) \\
& \times\left(K R^{1 / 2}-G P_{2} \bar{C}^{T} R^{-1 / 2}\right)^{T} .
\end{aligned}
$$

Noticing the expression of $K=G P_{2} \bar{C}^{T} R^{-1}+L U R^{-1 / 2}$ in (45) and the fact that $U U^{T}=I$, we have

$$
\left(K R^{1 / 2}-G P_{2} \bar{C}^{T} R^{-1 / 2}\right)\left(K R^{1 / 2}-G P_{2} \bar{C}^{T} R^{-1 / 2}\right)^{T}=L L^{T} .
$$

Thus, it follows from (50), that the definition of the matrix $L\left(L \in \mathbb{R}^{2 n \times p}\right)$ and the inequality (42) that $\Psi_{22}=\Pi+L L^{T}<0$.

To this end, we can conclude that $\Psi<0$. Therefore, it follows from (46) that

$$
\begin{aligned}
\left(A_{n}+\Delta A_{f}\right) P_{f}\left(A_{n}+\Delta A_{f}\right)^{T}-P_{f} & +J P_{f} J^{T} \\
& \leq-W_{f}+\Psi<0
\end{aligned}
$$

which leads to (33). As discussed earlier in Section III (see Remark 3), we know that the state of the augmented system (25) is mean-square bounded, and there exists a symmetric positive semi-definite solution to (32). The first claim of this theorem is then proved.

Furthermore, subtract (32) from (51) to give

$$
\begin{aligned}
&\left(A_{n}+\Delta A_{f}\right)\left(P_{f}-X\right)\left(A_{n}+\Delta A_{f}\right)^{T} \\
&-\left(P_{f}-X\right)+J\left(P_{f}-X\right) J^{T} \leq \Psi<0
\end{aligned}
$$

which indicates again from Remark 3 that $P_{f}-X \geq 0$ and, therefore

$$
X_{e e}=[X]_{22} \leq\left[P_{f}\right]_{22}=P_{2} .
$$

This completes the proof of this theorem.

Remark 4: We know from Theorem 1 that, if the conditions of Theorem 1 are all met, and the positive definite solution $P_{2}>$ 0 satisfies

$$
\left[P_{2}\right]_{i i} \leq \alpha_{i}^{2}, \quad i=1,2, \ldots, 2 n
$$

then, the filter (16) determined by (44) and (45) will be such that: 1) the augmented system (25) is mean-square bounded; and 2) $\left[X_{e e}\right]_{i i}<\left[P_{2}\right]_{i i} \leq \alpha_{i}^{2}, i=1,2, \ldots, 2 n$. Hence, the design objective of variance-constrained robust filter with random sensor delays will be accomplished. Note that the existence of a positive definite solution to (41) implies the asymptotical Schur stability of system matrix $A$, and then, the Assumption 1 should hold. 
Remark 5: In the present filter design method, there exists much explicit freedom, such as the choices of the free parameters $Z \in \mathbb{R}^{2 n \times 2 n}$ in (44), $L\left(L \in \mathbb{R}^{n \times p}\right.$ satisfies $\left.\Pi+L L^{T}<0\right)$, the orthogonal matrix $U \in \mathbb{R}^{p \times p}$, etc. We could use the resulting freedom to achieve more expected performance constraints (e.g., the transient requirement and reliability behavior on the filtering process).

Before discussing the numerical algorithm, we first point out that (41) can be transformed into the following linear-matrix inequality (LMI) by using the Schur Complement Lemma (Lemma 4)

$$
\left[\begin{array}{cc}
A P_{1} A^{T}-P_{1}+\varepsilon M M^{T}+D_{1} W D_{1}^{T} & A P_{1} N^{T} \\
N P_{1} A^{T} & -\varepsilon I+N P_{1} N^{T} \\
& <0 .
\end{array}\right.
$$

The inequality (54), together with the inequality constraint

$$
-\varepsilon I+N P_{1} N^{T}<0
$$

are both linear on $\varepsilon>0$ and $P_{1}>0$.

Hence, we can employ the standard LMI techniques in [5] to solve (41) and (55) for $\varepsilon>0$ and $P_{1}>0$, subjected to the linear constraint (43). Note that due to the special structures of $A, M$, $N, D_{1}$, and $W,(43)$ is not very restrictive as there are many zero entries. This will be illustrated in the numerical example. After $\varepsilon$ and $P_{1}$ are obtained, the inequality (42) becomes a standard Riccati-like matrix inequality, and some related solving algorithms can be found in [12], etc.

Summing up the results obtained, we have the following corollary.

Corollary 1: If there exist a positive scalar $\varepsilon>0$ and two positive definite matrices $P_{1}>0, P_{2}>0$ such that the LMIs (54) and (55), the (43), and the matrix Riccati inequality (42) hold, and $P_{2}>0$ satisfies $\left[P_{2}\right]_{i i} \leq \alpha_{i}^{2}(i=1,2, \ldots, 2 n$. $)$, then, the filter (16) determined by (44)-(45) will achieve the desired robust filtering performance for uncertain systems with random sensor delay.

\section{NUMERICAL EXAMPLE}

In this section, we demonstrate the theory developed in this note by means of a simple example. Consider the linear uncertain discrete-time stochastic system (1)-(3) with parameters given by

$$
\begin{aligned}
\tilde{A} & =\left[\begin{array}{cc}
0.2 & 0.05 \\
-0.02 & 0.3
\end{array}\right] \\
\tilde{C} & =\left[\begin{array}{cc}
10 & 0 \\
0 & 10
\end{array}\right] \\
\tilde{M} & =\left[\begin{array}{cc}
0.03 & -0.01 \\
0.02 & 0.06
\end{array}\right] \\
\tilde{N} & =\left[\begin{array}{cc}
0.05 & 0 \\
0 & 0.05
\end{array}\right] \\
W & =\left[\begin{array}{cc}
0.2 & 0 \\
0 & 0.2
\end{array}\right] \\
\tilde{V} & =\left[\begin{array}{cc}
0.1 & 0 \\
0 & 0.1
\end{array}\right]
\end{aligned}
$$

and the probability for complete observation (without random sensor delay) is assumed to be $\bar{\gamma}=0.9$.

The purpose of this example is to design the filter parameters $G$ and $K$ such that, for all admissible perturbations $\Delta A$, the augmented system (25) is mean-square bounded, and the steadystate error covariance $X_{e e}$ satisfies

$$
\begin{aligned}
& {\left[X_{e e}\right]_{11} \leq 3.5, \quad\left[X_{e e}\right]_{22} \leq 10} \\
& {\left[X_{e e}\right]_{33} \leq 3.5, \quad\left[X_{e e}\right]_{44} \leq 10}
\end{aligned}
$$

Solving the LMIs (54)-(55) for $\varepsilon, P_{1}$ (subject to the linear constraint (43)), and then the Riccati-like matrix inequality (42) for $P_{2}$, we obtain

$$
\begin{aligned}
\varepsilon & =8.8286 \\
P_{1} & =1.0 e+003 *\left[\begin{array}{cccc}
1.0970 & 0.0137 & 0.2201 & 0.0605 \\
0.0137 & 1.1541 & -0.0178 & 0.3460 \\
0.2201 & -0.0178 & 2.1470 & 0.0137 \\
0.0605 & 0.3460 & 0.0137 & 2.2041
\end{array}\right] \\
P_{2} & =\left[\begin{array}{cccc}
3.1522 & 0.1230 & 0.6361 & 0.4635 \\
0.1230 & 8.8239 & -0.0416 & 2.6467 \\
0.6361 & -0.0416 & 3.2522 & 0.1230 \\
0.4635 & 2.6467 & 0.1230 & 8.9239
\end{array}\right] .
\end{aligned}
$$

One of the filter parameters $G$ is calculated from (44) as follows:

$$
G=\left[\begin{array}{cccc}
0.2052 & 0.0500 & -0.0253 & -0.0016 \\
-0.0200 & 0.3178 & 0.0148 & -0.0584 \\
1.0000 & 0 & 0 & 0 \\
0 & 1.0000 & 0 & 0
\end{array}\right]
$$

To obtain another parameter $K$, we choose $L$ meeting $\Pi+$ $L L^{T}<0$ and select the orthogonal matrix $U$ as follows:

$$
\begin{aligned}
\text { Case 1: } L & =\left[\begin{array}{cc}
0.3 & 0 \\
0 & 0.3 \\
0 & 0 \\
0 & 0
\end{array}\right] \\
U & =I_{2} \\
\text { Case 2: } L & =\left[\begin{array}{cc}
0.2 & 0 \\
0 & 0.2 \\
0.2 & 0 \\
0 & 0.2
\end{array}\right] \\
U & =-I_{2} .
\end{aligned}
$$

Then, it follows from (45) that

$$
\begin{aligned}
& \text { Case } 1: K=\left[\begin{array}{cc}
0.0017 & 0 \\
-0.0000 & 0.0017 \\
0.0003 & 0.0001 \\
0 & 0.0009
\end{array}\right] \\
& \text { Case } 2: K=\left[\begin{array}{cc}
-0.0011 & 0.0001 \\
0 & -0.0009 \\
-0.0008 & 0.0001 \\
0 & -0.0002
\end{array}\right] \text {. }
\end{aligned}
$$

It is not difficult to verify that the specified mean square boundedness as well as the steady-state error variance constraint are achieved. 


\section{CONCLUSION}

In this paper, the linear filtering problem has been considered for parameter uncertain discrete-time stochastic systems randomly varying sensor delay. This problem has been tackled by assigning an upper bound to the steady-state error covariance, and by parameterizing the set of all filter gains that could achieve such an upper bound. It has been shown that the problem is solvable if several linear matrix inequalities or Riccati-like matrix inequalities have positive definite solutions. In particular, the characterization of the desired filter gains has been given in terms of some "free" parameters, and much design flexibility have been offered, which could be utilized to achieve more expected performance requirements. A numerical example has been provided to illustrate the effectiveness of the proposed design approach.

\section{REFERENCES}

[1] R. G. Agniel and E. I. Jury, "Almost sure boundedness of randomly sampled systems," SIAM J. Contr., vol. 9, pp. 372-384, 1971.

[2] A. Ben-Israel and T. N. E. Greville, Generalized Inverses: Theory and Applications. New York: Wiley, 1974.

[3] W. L. DeKoning, "Optimal estimation of linear discrete time systems with stochastic parameters," Automatica, vol. 20, pp. 113-115, 1984.

[4] M. Fu, C. E. de Souza, and Z.-Q. Luo, "Finite-horizon robust Kalman filter design," IEEE Trans. Signal Processing, vol. 49, pp. 2103-2112, Sept. 2001.

[5] P. Gahinet, A. Nemirovsky, A. J. Laub, and M. Chilali, LMI Control Toolbox: for Use With Matlab. Natick, MA: MATHWorks, 1995.

[6] H. Li and M. Fu, "Linear matrix inequality approach to robust $H_{\infty}$ filtering," IEEE Trans. Signal Processing, vol. 45, pp. 2338-2350, Sept. 1997.

[7] M. Malek-Zavarei and M. Jamshidi, Time-Delay Systems: Analysis, Optimation and Application. Amsterdam, The Netherlands: North-Holland, 1987.
[8] S. I. Niculescu, E. I. Verriest, L. Dugard, and J. M. Dion et al., "Stability and robust stability of time-delay systems: A guided tour," in Stability and Control of Time-Delay Systems, L. Dugard et al., Eds. Berlin, Germany: Springer, 1998, vol. 228, pp. 1-71.

[9] R. M. Palhares, C. E. de Souza, and P. L. Dias Peres, "Robust $H_{\infty}$ filtering for uncertain discrete-time state-delayed systems," IEEE Trans. Signal Processing, vol. 49, pp. 1696-1703, Aug. 2001.

[10] A. W. Pila, U. Shaked, and C. E. de Souza, " $H_{\infty}$ filtering for continuous linear system with delay," IEEE Trans. Automat. Contr., vol. 44, pp. 1412-1417, July 1999.

[11] A. Ray, "Output feedback control under randomly varying distributed delays," J. Guidance, Contr. Dyn., vol. 17, no. 4, pp. 701-711, 1994.

[12] A. Saberi, P. Sannuti, and B. M. Chen, $\mathrm{H}_{2}$ Optimal Control. London, U.K.: Prentice-Hall, 1995.

[13] U. Shaked, L. Xie, and Y. C. Soh, "New approaches to robust minimum variance filter design," IEEE Trans. Signal Processing, vol. 49, pp. 2620-2629, Nov. 2001.

[14] R. F. Stengel, Stochastic Optimal Control: Theory and Application. New York: Wiley, 1986.

[15] Y. Wang, L. Xie, and C. E. de Souza, "Robust control of a class of uncertain nonlinear systems," Syst. Contr. Lett., vol. 19, pp. 139-149, 1992.

[16] Z. Wang and B. Huang, "Robust $H_{2} / H_{\infty}$ filtering for linear systems with error variance constraints," IEEE Trans. Signal Processing, vol. 48, pp. 2463-2467, Aug. 2000

[17] Z. Wang, B. Huang, and P. Huo, "Sampled-data filtering with error covariance assignment," IEEE Trans. Signal Processing, vol. 49, pp. 666-670, Mar. 2001.

[18] Z. Wang and H. Qiao, "Robust filtering for bilinear uncertain stochastic discrete-time systems," IEEE Trans. Signal Processing, vol. 50, pp. 560-567, Mar. 2002.

[19] Z. Wang and F. Yang, "Robust filtering for uncertain linear systems with delayed states and outputs," IEEE Trans. Circuits Syst. I, vol. 49, pp. 125-130, Jan. 2002.

[20] Z. Wang, G. Shan, and Z. Guo, "A new approach to the study of statistical law of missing data for laser distance-measured systems," Fire Contr. Command Contr., vol. 22, pp. 25-28, 1997.

[21] E. Yaz and R. E. Skelton, "Continuous and discrete state estimation with error covariance assignment," in Proc. IEEE Conf. Decision Control, Brighton, U.K., 1991, pp. 3091-3092.

[22] E. Yaz and A. Ray, "Linear unbiased state estimation for random models with sensor delay," in Proc. IEEE Conf. Decision Control, Kobe, Japan, 1996, pp. 47-52. 